\title{
Mesenchymal stromal cell therapy in COPD: from bench to bedside
}

\author{
This article was published in the following Dove Press journal: \\ International Journal of COPD \\ 16 October 2017 \\ Number of times this article has been viewed
}

\author{
Mariana A Antunes ${ }^{1,2}$ \\ José Roberto Lapa e Silva ${ }^{3}$ \\ Patricia RM Roccol,2 \\ 'Laboratory of Pulmonary \\ Investigation, Carlos Chagas Filho \\ Institute of Biophysics, Federal \\ University of Rio de Janeiro, Rio de \\ Janeiro (UFRJ), RJ, Brazil; ${ }^{2}$ National \\ Institute of Science and Technology \\ for Regenerative Medicine, Rio \\ de Janeiro, RJ, Brazil; ${ }^{3}$ Institute of \\ Thoracic Medicine, Clementino Fraga \\ Filho University Hospital, Federal \\ University of Rio de Janeiro (UFRJ), \\ Rio de Janeiro, RJ, Brazil
}

\begin{abstract}
COPD is the most frequent chronic respiratory disease and a leading cause of morbidity and mortality. The major risk factor for COPD development is cigarette smoke, and the most efficient treatment for COPD is smoking cessation. However, even after smoking cessation, inflammation, apoptosis, and oxidative stress may persist and continue contributing to disease progression. Although current therapies for COPD (primarily based on anti-inflammatory agents) contribute to the reduction of airway obstruction and minimize COPD exacerbations, none can avoid disease progression or reduce mortality. Within this context, recent advances in mesenchymal stromal cell (MSC) therapy have made this approach a strong candidate for clinical use in the treatment of several pulmonary diseases. MSCs can be readily harvested from diverse tissues and expanded with high efficiency, and have strong immunosuppressive properties. Preclinical studies have demonstrated encouraging outcomes of MSCs therapy for lung disorders, including emphysema. These findings instigated research groups to assess the impact of MSCs in human COPD/emphysema, but clinical results have fallen short of expectations. However, MSCs have demonstrated a good adjuvant role in the clinical scenario. Trials that used MSCs combined with another, primary treatment (eg, endobronchial valves) found that patients derived greater benefit in pulmonary function tests and/or quality of life reports, as well as reductions in systemic markers of inflammation. The present review summarizes and describes the more recent preclinical studies that have been published about MSC therapy for COPD/emphysema and discusses what has already been applied about MSCs treatment in COPD patients in the clinical setting.
\end{abstract}

Keywords: emphysema, mesenchymal stromal cells, inflammation, remodeling, repair

\section{Introduction}

The Global Initiative for Chronic Obstructive Lung Disease (GOLD) defines COPD as a "common, preventable and treatable disease that is characterized by persistent respiratory symptoms and airflow limitation that is due to airway and/or alveolar abnormalities usually caused by significant exposure to noxious particles or gases". COPD is the fourth leading cause of death worldwide and is projected to be the third by 2020, because of increasing exposure to COPD risk factors and population aging. ${ }^{2,3}$

Chronic airflow limitation is the main feature of COPD, caused by a combination of small-airway disease (bronchitis) and parenchymal destruction (emphysema), the relative contributions of which vary from person to person. Emphysema has been classically defined as a destruction of the gas-exchange surfaces in the lung, and chronic bronchitis, and as the presence of cough and sputum production for at least 3 months each in 2 consecutive years. Nevertheless, in the latest COPD guidelines, the terms "bronchitis" and "emphysema" are no longer used, because these limited conceptual definitions may underestimate the number of diagnosed patients. It is 
relevant to recognize that chronic respiratory symptoms precede the development of airflow limitation and may exist in individuals with normal pulmonary function; for instance, a significant number of smokers with no airflow limitation present structural evidence of lung disease, represented by emphysema, airway wall thickening, and gas trapping. ${ }^{1}$

Current treatments for COPD are based on an individualized assessment to reduce both symptoms and future risk of exacerbations. Available pharmacologic approaches are mainly based on anti-inflammatory drugs, including corticosteroids, theophylline, and bronchodilators. These only act to minimize airflow limitation and acute exacerbations and do improve the quality of life of patients with COPD; however, there is no available therapy that prevents disease progression or reduces mortality. Thus, it is urgent to develop new therapies for COPD.

Mesenchymal stromal cells (MSCs) have been widely investigated in respiratory diseases, ${ }^{4}$ and are strong candidates to treat destructive disorders such as COPD/emphysema. ${ }^{5}$ MSCs are multipotent, non-hematopoietic progenitors that may be harvested from several tissues and expanded in vitro with high efficiency. Their intense immunosuppressive properties may be essential for successful autologous and heterologous transplantation. Preclinical studies have shown benefits of MSCs in several pulmonary conditions, including asthma, ${ }^{6} \mathrm{COPD} / \mathrm{emphysema},{ }^{5}$ acute respiratory distress syndrome, ${ }^{7}$ lung fibrosis, ${ }^{8}$ and pulmonary hypertension models. ${ }^{9}$ These positive results encouraged several translational studies of MSCs for lung diseases that have yielded some promising outcomes, including for COPD.

MSC therapy of lung diseases is a wide-ranging subject. In this review, we intended to focus on summarizing the most recent research into MSC therapy in experimental models of pulmonary emphysema, the main therapeutic mechanisms of MSCs that have been elucidated by these studies, and what ongoing clinical trials of MSCs in COPD have brought in terms of hopeful outcomes.

\section{MSC therapy in COPD: the preclinical scenario}

In 2006, Shigemura et al published the first study involving MSC transplantation in an experimental model of pulmonary emphysema, ${ }^{10}$ which left many issues unanswered, such as the following: 1) which is the best MSCs lineage to treat emphysema; 2) which is the best route of administration; 3 ) whether single or multiple injections are superior; and 4) whether MSCs are protagonists or best as adjuvant therapy. Eleven years later, there is still no definitive answer for any of these questions, only evidence of how far we remain from a definitive protocol for MSC therapy in COPD (Table 1).

MSCs may be harvested from several adult tissues. In emphysema models, different sources of MSCs have been tested, including adipose tissue (AD), , ${ }^{5,13}$ lung, ${ }^{5,14}$ umbilical cord, ${ }^{15}$ human tubal tissue, ${ }^{16}$ amniotic fluid, ${ }^{17}$ and bone marrow (BM), ${ }^{5,18-21}$ which is the most widely used source. MSCs may present different phenotypes depending on their site of origin, resulting in differences in immunogenicity, anti-inflammatory and regenerative ability, and expansibility in culture..$^{22,23}$ These differences might influence the impact of MSCs in vivo. In the same experimental model of elastase emphysema, BM-derived, AD-derived, and pulmonary tissue (L)-derived MSCs were evaluated comparatively. All 3 types revealed some similar beneficial effects on cardiopulmonary function and/or histology, contributing to improvement of the mean linear intercept, neutrophil infiltration, collagen deposition, and elastic fiber synthesis; however, some results were only achieved with specific types of MSCs, especially bone marrow mesenchymal stromal cells (BM-MSCs), which seem to be more effective in inducing macrophage polarization into a more anti-inflammatory phenotype. The existence of an "environmental-niche memory" could explain this particular effect of BM-MSCs, while AD-MSCs, which are described as having an "epithelial" commitment, ${ }^{24}$ are not as effective in modulating macrophage polarization. BM-MSCs also demonstrate great effect in reverting pulmonary arterial hypertension induced by emphysema and in reducing epithelial and endothelial cell apoptosis in the lung in 2 experimental models (elastase and cigarette smoke [CS]). ${ }^{5,20}$ The engraftment rates of MSCs are low. Paracrine action is the main mechanism explaining their effects, regardless of source. Evidence reveals that MSCs from several sources (BM, $,^{5,20} \mathrm{AD},{ }^{5,13,25}$ pulmonary tissue, ${ }^{5,14}$ tubal tissue, ${ }^{16}$ amniotic fluid ${ }^{17}$ ) act by modulating inflammatory and reparative mediators, without necessarily being present at the site of lesion.

As far as emphysema models are concerned, 2 main delivery routes have been tested: systemic (intravenous, intraperitoneal) and local (intratracheal, intrabronchial, intrapleural, intranasal). Due to its cardiovascular and muscle weakness components, COPD should not be considered an exclusively pulmonary disorder. Besides, in terms of translational research, MSCs should be associated with less-invasive procedures and less contamination risks, which would support the systemic delivery. This has been the preferred route in the preclinical setting and has been used 
Table I Preclinical studies of MSC therapy in rodent models of emphysema

\begin{tabular}{|c|c|c|c|c|c|c|c|c|}
\hline First author & Year & Animal & $\begin{array}{l}\text { COPD } \\
\text { model }\end{array}$ & $\begin{array}{l}\text { MSC } \\
\text { source }\end{array}$ & $\begin{array}{l}\text { Delivery } \\
\text { route }\end{array}$ & Dose & Regimen & Main parameters evaluated \\
\hline $\begin{array}{l}\text { Shigemura } \\
\text { et al }{ }^{10}\end{array}$ & 2006 & Rat & PPE & AD-MSCs & IV & $5 \times 10^{7}$ & Single dose & $\begin{array}{l}\text { HGF, TUNEL, PCNA, RAC index, } \\
\text { vascular density, } \mathrm{PaO}_{2}\end{array}$ \\
\hline Zhen et $\mathrm{a}^{47}$ & 2008 & Rat & Papain & BM-MSCs & IV & $4 \times 10^{6}$ & Single dose & Lm, TUNEL \\
\hline Zhen et $\mathrm{al}^{48}$ & 2010 & Rat & Papain & BM-MSCs & IV & $4 \times 10^{6}$ & Single dose & Lm, TUNEL, caspase-3, VEGF \\
\hline Huh et $\mathrm{al}^{20}$ & 2011 & Rat & CS & BM-MSCs & IV & $6 \times 10^{5}$ & Single dose & $\mathrm{Lm}$, apoptosis \\
\hline Katsha et $\mathrm{al}^{49}$ & 2011 & $\begin{array}{l}\text { Mouse } \\
(\mathrm{C} 57 \mathrm{~B} / 6)\end{array}$ & PPE & BM-MSCs & IT & $5 \times 10^{5}$ & Single dose & Lm, destructive index, IL-I $\beta$, HGF, EGF \\
\hline $\begin{array}{l}\text { Schweitzer } \\
\text { et } \mathrm{al}^{25}\end{array}$ & 2011 & Rat & CS & $\begin{array}{l}\text { Human } \\
\text { AD-MSCs }\end{array}$ & IV & $3 \times 10^{5}$ & Single dose & $\begin{array}{l}\text { Lung macrophage, PMN, caspase- } 3 \text {, } \\
\text { lung volume, alveolar surface area }\end{array}$ \\
\hline Guan et $\mathrm{al}^{36}$ & 2013 & Rat & CS & BM-MSCs & IT & $6 \times 10^{6}$ & Single dose & $\begin{array}{l}\text { Lm, TUNEL, caspase-3, VC, FEV } \\
\text { MMP-9, MMP-12, TGF- } \beta I \text {, VEGF }\end{array}$ \\
\hline $\begin{array}{l}\text { Antunes } \\
\text { et } \mathrm{al}^{5}\end{array}$ & 2014 & $\begin{array}{l}\text { Mouse } \\
(\mathrm{C} 57 \mathrm{BI} / 6)\end{array}$ & PPE & $\begin{array}{l}\text { BM-MSCs, } \\
\text { AD-MSCs, } \\
\text { LD-MSCs }\end{array}$ & IV/IT & $1 \times 10^{5}$ & Single dose & $\begin{array}{l}\mathrm{Lm} \text {, normal and hyperinflated alveolar } \\
\text { areas, neutrophils, echocardiography, } \\
\text { TUNEL, Lm, collagen, and elastic fibers }\end{array}$ \\
\hline Li et $\mathrm{al}^{40}$ & 2014 & Rat & CS & $\begin{array}{l}\text { Human } \\
\text { iPS-MSCs/ } \\
\text { BM-MSCs }\end{array}$ & IV & $3 \times 10^{6}$ & Two doses & Lm, collagen \\
\hline Li et al ${ }^{17}$ & 2014 & Rat & CS + LPS & AFD-MSCs & IT & $4 \times 10^{5}$ & Two doses & Lm, TUNEL \\
\hline $\begin{array}{l}\text { Tibboel } \\
\text { et al }{ }^{21}\end{array}$ & 2014 & $\begin{array}{l}\text { Mouse } \\
(\mathrm{C} 57 \mathrm{Bl} / 6)\end{array}$ & PPE & BM-MSCs & IT/IV & $5 \times 10^{5} / 1 \times 10^{5}$ & Single dose & $\begin{array}{l}\mathrm{Lm} \text {, dynamic compliance, mean forced } \\
\text { expiratory flow }\end{array}$ \\
\hline Zhao et a ${ }^{50}$ & 2014 & Rat & $\mathrm{CS}+\mathrm{LPS}$ & BM-MSCs & IV & $5 \times 10^{6}$ & Single dose & $\begin{array}{l}\text { Mean alveoli number, pulmonary } \\
\text { alveolar area }\end{array}$ \\
\hline Chen et a $\left.\right|^{18}$ & 2015 & $\begin{array}{l}\text { Mouse } \\
(\mathrm{C} 57 \mathrm{~B} / 6)\end{array}$ & PPE & BM-MSCs & IV & Unknown & Single dose & $\begin{array}{l}\text { Lm, VEGF, HSP70, whole body } \\
\text { plethysmography }\end{array}$ \\
\hline Gu et $\mathrm{al}^{19}$ & 2015 & Rat & CS & BM-MSCs & IT & $6 \times 10^{6}$ & Single dose & $\begin{array}{l}\text { Lm, COX2, PGE2, IL-6, IL-10, } \\
\text { inflammation score }\end{array}$ \\
\hline Kim et al ${ }^{15}$ & 2015 & $\begin{array}{l}\text { Mouse } \\
(\mathrm{C} 57 \mathrm{BI} / 6)\end{array}$ & PPE & $\begin{array}{l}\text { Human } \\
\text { CBD-MSCs }\end{array}$ & IV & $1.10^{4} / 2.5 .10^{4} / 5.10^{4} / 1.10^{5}$ & Single dose & Lm, VEGF \\
\hline Peron et $\mathrm{al}^{16}$ & 2015 & $\begin{array}{l}\text { Mouse } \\
(\mathrm{C} 57 \mathrm{BI} / 6)\end{array}$ & $\begin{array}{l}\mathrm{CS}+ \\
\text { irradiation }\end{array}$ & $\begin{array}{l}\text { Human } \\
\text { tubal MSCs }\end{array}$ & IP/IN & $1 \times 10^{6}$ & Two doses & $\begin{array}{l}\text { BALF total cell count, neutrophil } \\
\text { count, airway mucus, collagen }\end{array}$ \\
\hline Hong et $\mathrm{al}^{12}$ & 2016 & $\begin{array}{l}\text { Mouse } \\
(\mathrm{C} 57 \mathrm{BI} / 6)\end{array}$ & $\mathrm{PPE} / \mathrm{CS}$ & $\begin{array}{l}\text { Human } \\
\text { AD-MSCs }\end{array}$ & IV & $1 \times 10^{5}$ & Single dose & Lm, VEGF, HGF, FGF2, caspase-3/7 \\
\hline $\begin{array}{l}\text { Kennelly } \\
\text { et } \mathrm{al}^{35}\end{array}$ & 2016 & $\begin{array}{l}\text { NOD-SCID } \\
\text { IL-2rgull mice }\end{array}$ & PPE & $\begin{array}{l}\text { Human } \\
\text { BM-MSCs }\end{array}$ & IV & $5 \times 10^{5}$ & Single dose & $\begin{array}{l}\text { Lm, alveoli number, IL-I } \beta, \text { IL-6, } \\
\text { collagen deposition, TUNEL }\end{array}$ \\
\hline Cho et al" & 2017 & $\begin{array}{l}\text { Mouse } \\
(\mathrm{C} 57 \mathrm{BI} / 6)\end{array}$ & PPE & $\begin{array}{l}\text { Human } \\
\text { AD-MSCs }\end{array}$ & IP & $1 \times 10^{5}$ & Single dose & $\begin{array}{l}\text { Lm, BAX, BcI2, FGF2, VEGF, HGF, } \\
\text { MMP2, MMPI2, TIMPI, SLPI }\end{array}$ \\
\hline
\end{tabular}

Abbreviations: AD, adipose tissue; AFD, amniotic fluid; BALF, bronchoalveolar lavage fluid; BAX, apoptosis regulator (also known as bcl-2-like protein 4); Bcl2, antiapoptotic regulator; BM, bone marrow; CBD, umbilical cord blood; COX2, cyclooxygenase 2; CS, cigarette smoke; EGF, epidermal growth factor; FEV, forced expiratory volume in one second; FGF2, fibroblast growth factor 2; HGF, hepatocyte growth factor; HSP70, heat shock protein; IL, interleukin; IN, intranasal; iPS, induced pluripotent stem cells; IT, intratracheal; IP, intraperitoneal; IV, intravenous; LD, lung tissue; Lm, mean linear intercept; LPS, lipopolysaccharide; MMP, matrix metalloproteases; MSC, mesenchymal stromal cell; NOD-SCID IL-2rgull mice, NOD/SCID/II-2 receptor $\gamma$-chain null mice that lack an adaptive immune system, facilitating the successful administration of human MSCs; SLPI, human leukocyte elastase inhibitor; $\mathrm{PaO}_{2}$, arterial oxygen partial pressure; PCNA, proliferating cell nuclear antigen; PGE2, prostaglandin E2; PMN, polymorphonuclear cells; PPE, porcine pancreatic elastase; RAC, radial alveolar count; TGF- $\beta$ I, transforming growth factor beta I; TIMPI, metalloproteases inhibitor; TUNEL, Terminal deoxynucleotidyl transferase dUTP nick end labeling; VC, vital capacity; VEGF, vascular endothelial growth factor.

successfully. However, to reach the body circulation after transplant at an extent sufficient to act in both the pulmonary and extrapulmonary compartments, and considering severe models of emphysema, the number of MSCs injected would have to be very high, which could preclude reproduction in experimental studies due to the restrictions of administering large cell counts to small animals. For this reason, local administration routes became strong candidates for MSC treatment of emphysema. MSCs act independently of the route of administration, but evidence has demonstrated that intravenous injection is more effective in achieving immunomodulatory effects (eg, induction of macrophage polarization and endothelial cell proliferation, production of vascular endothelial growth factor [VEGF]), while the local route (intratracheal) yields more intense reparative mechanisms (eg, reduction in lung hyperinflation and fibrosis) in a chronic model of elastase-induced emphysema. ${ }^{5}$

A wide range of MSCs doses $\left(10^{4}\right.$ up to $\left.6 \times 10^{6}\right)$ have been used to treat emphysema in research settings. The choice of optimal dose is a critical part of study design and must take 
into account important issues, including the route of administration, the animal species to be used, the dosage regimen (single or multiple doses), and the degree of emphysema. These different factors make it difficult to determine the best dose and hinder comparison across studies. In the elastaseinduced emphysema model, a dose-response curve was designed using 4 different doses $\left(10^{4}, 2.5 \times 10^{4}, 5 \times 10^{4}\right.$, and $\left.10^{5}\right)$ of human umbilical cord blood-derived MSCs, based on their effects on the mean linear intercept. ${ }^{15}$ The authors of this study suggested that the optimal dose to treat emphysema as in their model is $5 \times 10^{4} \mathrm{MSCs}$, but there was only slight variation among the tested doses and effect on the benchmark outcome (mean linear intercept) to assume that this is indeed the optimal dose, from which the rest of the study was designed. ${ }^{15}$ To date, no further study has been conducted to determine the optimal dose considering all the critical points mentioned in this paragraph.

Most research uses MSCs as a single treatment when evaluating their potential impact on emphysema. These studies have demonstrated that MSCs actively contribute to the repair of lung damage induced by experimental emphysema. Although MSC administration has shown to be safe in patients with COPD, clinical trials in this field have not demonstrated robust therapeutic effects, such as pulmonary function improvement or mortality reduction. ${ }^{26,27}$ Therefore, new advanced methods to increase MSC potency, such as increasing differentiation efficiency and/or paracrine effects and achieving more persistent engraftment, may be required. Modern research lines combine stromal cell therapy with a specific mediator or a classic treatment to potentiate or improve the benefits of MSCs on the recovery of pulmonary tissue and function. ${ }^{11,12,16}$

The peroxisome proliferator-activated receptor (PPAR)- $\gamma$ agonist pioglitazone is known to enhance adipocyte differentiation. PPAR- $\gamma$ is also expressed in MSCs, and evidence suggests that pioglitazone may change MSC phenotype during cell differentiation, enhance differentiation, and improve the therapeutic effects of MSCs in the heart. ${ }^{28}$ Pioglitazone has been suggested to potentiate epithelial cell growth and proliferation rates in vitro, as well as to increase VEGF production by these cells. In 1 study of AD-MSCs pretreated or not with pioglitazone and transplanted into mice with elastase-induced or CS-induced emphysema, ${ }^{12}$ pretreated MSCs were more efficient in reducing mean linear intercept compared with the naïve MSCs; however, only in the cigarette-smoke model did pioglitazone-treated MSCs led to a more intense modulation of growth factors involved in the repair of emphysematous lungs, including
VEGF, hepatocyte growth factor (HGF), and fibroblast growth factor (FGF)-2. ${ }^{12}$

Low-level laser (LLL) has been suggested as an interesting candidate therapy to boost the overall response to MSCs in emphysema. LLL is noninvasive, safe, and economical, and it seems to promote stem-cell proliferation in vitro. ${ }^{29}$ Furthermore, evidence shows that alveolar macrophages (a key cell type in COPD) irradiated with LLL may exhibit increased cyclic adenosine monophosphate synthesis, which leads to a reduction of nuclear factor-kappa $B$ activation and, consequently, interleukin (IL)-6 and IL-1 secretion in the context of lung inflammation. ${ }^{30}$ Recently, human tubal MSCs (htMSCs) and LLL were successfully combined to treat a murine model of CS-induced emphysema. ${ }^{16}$ Combined administration with LLL potentiated some of the beneficial effects of htMSCs, especially the reduction of cellular infiltration, mucus secretion, and collagen deposition in the lungs. ${ }^{16}$

Optimization of culture conditions has also been attempted as another strategy to improve MSC functions. MSCs have been classically cultured in a 2D monolayer using culture plates. Nevertheless, in 2D cultures, MSCs may lose their stemness properties (replication potential, differentiation capacity) which does not occur in vivo. For this reason, tests have begun on 3D-spheroid cultures (self-assembled group of cells). The spheroid formation prevents apoptosis and facilitates differentiation, due to the conservation of cellcell interactions that are important for survival and colony formation. ${ }^{31}$ Recently, the effect of aggregated spheroid AD-MSCs was compared with that of dissociated ADMSCs in a murine model of elastase-induced emphysema. ${ }^{11}$ Spheroid AD-MSCs presented higher protein expression of the antiapoptotic mediator Bcl-2 and lower expression of the apoptotic regulator BAX compared with dissociated AD-MSCs. In addition, spheroid AD-MSCs exhibit better therapeutic performance in elastase emphysema compared with dissociated AD-MSCs (greater reduction in mean linear intercept, higher expression of FGF-2 in lung tissue, greater reduction in matrix metalloproteinase [MMP]-2 activity and MMP-12 gene expression, and a greater increase in gene expression of tissue inhibitor of metalloproteinase-1 and secretory leukocyte protease inhibitor). ${ }^{11}$

\section{MSC therapy in COPD: revealed mechanisms}

Tracking studies reveal that most MSCs disappear within 1 day after injection. ${ }^{13,32}$ There is a consensus that MSCs may differentiate into bone, $\mathrm{AD}$, and cartilage in vitro, but that they rarely differentiate into tissue-resident cells in the lungs 
(eg, epithelial cells), whereas they effectively act by paracrine mechanisms and through immunomodulatory functions in vivo. ${ }^{33}$ MSCs may deliver some signals to host cells, inducing a regenerative mechanism against alveolar destruction in COPD lungs. Gene profiling studies in pulmonary cells after transplant may be an important tool for understanding the regenerative mechanisms of MSCs in injured tissue. In a 6-month CS-induced emphysema model, changes in the gene expression profile of mouse lungs were evaluated over time (on days 1, 4, and 14) following systemic injection of human cord blood-derived MSCs (hCB-MSCs). ${ }^{34}$ This report determined that hCB-MSCs induce time-dependent molecular changes in the lung, with regulation of immune responses, oxidative stress, and transcription soon after hCBMSC injection (on days 1 and 4), while blood vessel development and cell growth regulation predominated at a later stage (on day 14) compared to controls. This is consistent with evidence of a time-dependent effect of the protective role of MSCs. Although MSCs have been demonstrated as effective even when the structural changes of emphysema are already established, their potency is slightly reduced..$^{35}$ One of the main soluble factors released by MSCs and related to their anti-apoptotic effects is HGF. HGF knockdown in human BM-MSCs (generated through short hairpin RNA) led to reduction of cytoprotection (a high level of alveolar wall destruction) and minimal effects on lung tissue apoptosis in a model of elastase-induced emphysema when compared with control (non-knockdown) MSCs. ${ }^{35}$

MSC-mediated immunomodulation is one of the main mechanisms of action of these cells in emphysema and involves downregulation of several pro-inflammatory mediators (tumor necrosis factor- $\alpha$, IL-1 $1 \beta$, IL- 6 and monocyte chemoattractant protein-1) and proteases (MMP-9 and MMP-12), as well as upregulation of mediators such as transforming growth factor- $\beta 1$ and VEGF in lung tissue. ${ }^{36}$ A wealth of evidence supports a "vascular hypothesis" as a major feature of COPD pathophysiology. This theory is based on lower levels of VEGFA in COPD lungs (compared with those of healthy subjects) and partial cleavage of VEGFA by neutrophil elastase, leading to VEGFA fragments with altered activity and loss of signaling potential in endothelial cells. VEGFA is essential for endothelial cell survival, and its modulation plays a significant role in the pathophysiology of several lung disorders, including COPD and pulmonary hypertension. Reports have demonstrated that VEGFA may help prevent apoptosis and tissue injury in COPD; ${ }^{37}$ however, continued overexpression of VEGFA has many side effects. ${ }^{38,39}$ To avoid the complications associated with constitutive VEGFA expression, researchers have achieved conditional expression of VEGFA by MSCs under the regulation of a $c$-resveratrol ( $c$-RSV)-inducible heat shock protein (HSP)-70 promoter. ${ }^{18}$ HSP70 concentration is increased in the serum of COPD patients and has a linear relationship with disease severity. $c$-RSV, a phytochemical that indirectly activates HSP70 promoter activity, induces expression of antioxidant-related genes in HSP70-VEGFA-MSCs in vitro, including nuclear factor erythroid-2-related factor (Nrf-2) and heme oxygenase-1 (HO-1). The rapid decline in engrafted MSC counts after the first $24 \mathrm{~h}$ post-transplantation may also be related to exposure to toxic and oxidative microenvironments. HSP70-VEGFA-MSCs exhibited higher survival than control MSCs in a CS extract challenge, and combined treatment with $c$-RSV and engraftment of HSP-VEGFAMSCs promoted better therapeutic effects than mock control in elastase-induced emphysema: upregulation of antioxidant gene expression (Nrf-2, HO-1, and manganese-dependent superoxide dismutase) and reduced mean linear intercept. ${ }^{18}$

In addition to paracrine actions, MSCs interact with the immune system rather than through direct actions on the lung. MSCs modulate macrophages activity, reversing inflammation and lung dysfunction in emphysema. ${ }^{5,19}$ MSCs may induce alveolar macrophage polarization toward an anti-inflammatory phenotype (M2) while inhibiting proinflammatory phenotype (M1), contributing to improvement of lung inflammation and elastolysis. ${ }^{5}$ Consistent with this finding, another study reported a decrease in the percentage of CD68-positive pulmonary cells after MSC administration in CS-induced emphysema. Nevertheless, MSCs increased the percentage of IL-10-positive cells within the CD68positive population $\left(\mathrm{CD} 68^{+} \mathrm{IL}-10^{+}\right)$, which probably represents M2 macrophages. ${ }^{19}$ Moreover, MSCs may interact with alveolar macrophages via cell-to-cell contact and promote their reprogramming via the cyclooxygenase-2 (COX-2)/ prostaglandin (PG)E2 pathway. CS-mediated COX-2 upregulation leads to increased production of PGs, which play essential roles in the inflammatory cascade. MSC treatment reduces PGE2 production by CS-stimulated macrophages in vitro. COX-2 expression during inflammation is mediated by the family of mitogen-activated protein kinases (MAPKs), which includes extracellular signal-regulated kinase (ERK), c-Jun N-terminal kinase (JNK), and p38. However, the effect of MSCs on CS-induced COX-2 expression in macrophages occurs only via the p38 MAPK and ERK cascades, without involvement of the JNK pathway. ${ }^{19}$

CS-induced mitochondrial dysfunction plays a critical role in pulmonary emphysema. Recent reports demonstrate 
that MSCs may modulate lung epithelial and endothelial cells through mitochondrial transfer via formation of tunneling nanotubes (TNTs), improving host cell bioenergetics. ${ }^{40,41}$ TNTs are highly sensitive nanotubular structures, formed in vitro between cells, that optimize selective transfer of membrane vesicles and organelles ${ }^{42}$ and may mediate mitochondrial transfer from MSCs to epithelial cells. ${ }^{43}$ Co-culture of BM-MSCs or induced pluripotent stem cellderived MSCs (iPSC-MSCs) with a bronchial epithelial cell line (BEAS-2B) treated with CS medium (CSM) create an in vitro scenario most similar to clinical emphysema. In this context, TNT-like structures are observed between iPSCMSCs and BM-MSCs and BEAS-2B cells, as well as abundant presence of MSC-derived mitochondria in BEAS-2B cells within $24 \mathrm{~h}$, suggesting a mitochondrial transfer that is mediated by TNT formation. ${ }^{40}$ Although mitochondrial transfer seems to be a common mechanism used by MSCs, transfer intensity may be influenced by the MSC source that is chosen. A significantly higher mitochondrial transfer rate is observed in BEAS-2B cells with administration of iPSC-MSCs than with administration of BM-MSCs; this may be potentiated even further in the presence of CSM. ${ }^{40}$ Studies have shown that mitochondrial transfer from MSCs to alveolar type II epithelial cells (ATII) improves survival in a lipopolysaccharide (LPS)-induced pneumonia model through restoration of ATII bioenergetics. Extensive mitochondrial transfer from MSCs to macrophages as well as to resident cells has been demonstrated in an LPS-induced pneumonia model, both in vitro and in vivo. This mitochondrial transfer from MSCs to macrophages has been associated to increased phagocytic activity. However, to date, there is no evidence of mitochondrial transfer from MSCs and macrophages in the context of COPD.

\section{MSC therapy in COPD: clinical trials}

Positive outcomes in the preclinical scenario have encouraged clinical trials of MSC therapy in pulmonary emphysema. A search of the ClinicalTrials.gov database for articles published until June 2017, using specific keywords ("stromal cell" and COPD; "stem cell" and COPD; "stromal cell" and "emphysema"; "stem cell" and "emphysema"), yielded 17 studies examining the effects of MSCs on COPD or emphysema in human clinical trials (Table 2). Of these, only 4 have been completed and had data published in the PubMed database. Ongoing clinical trials of stem cell-based therapies in COPD include mainly AD-MSCs and BM-MSCs. The majority of these studies (7 of 11 ongoing trials) use ADMSCs transplantation, probably due to the fact that these

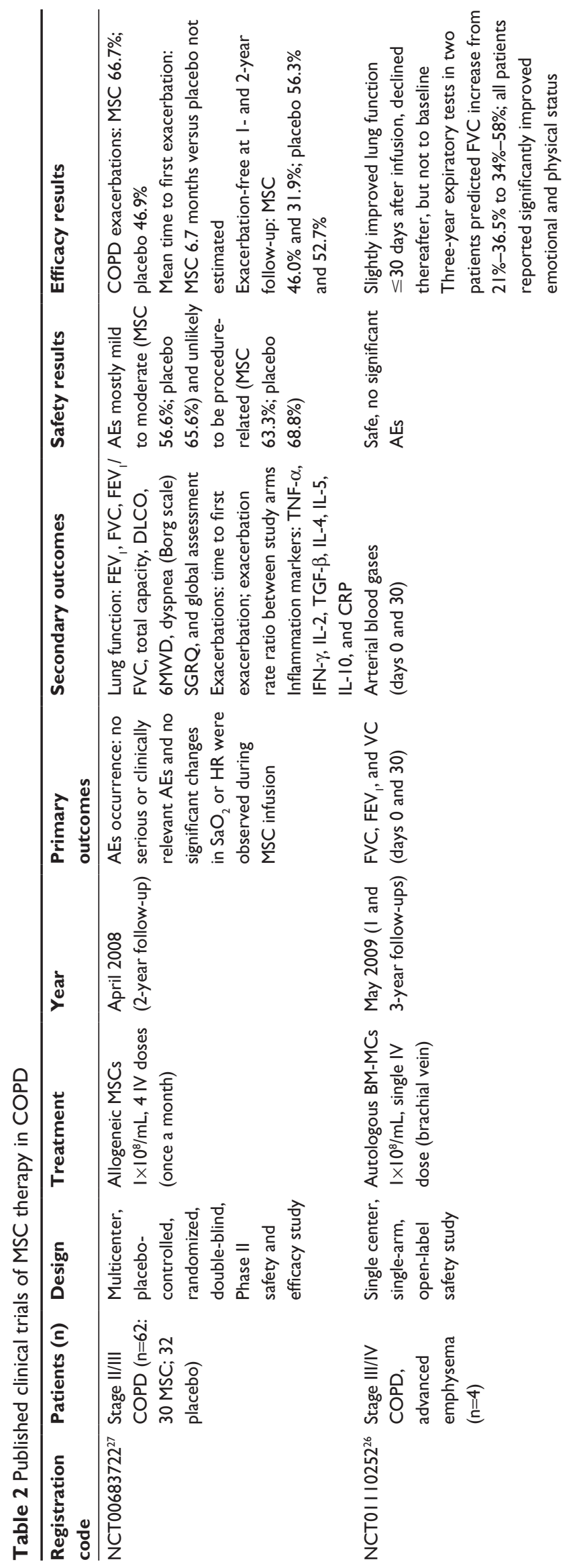




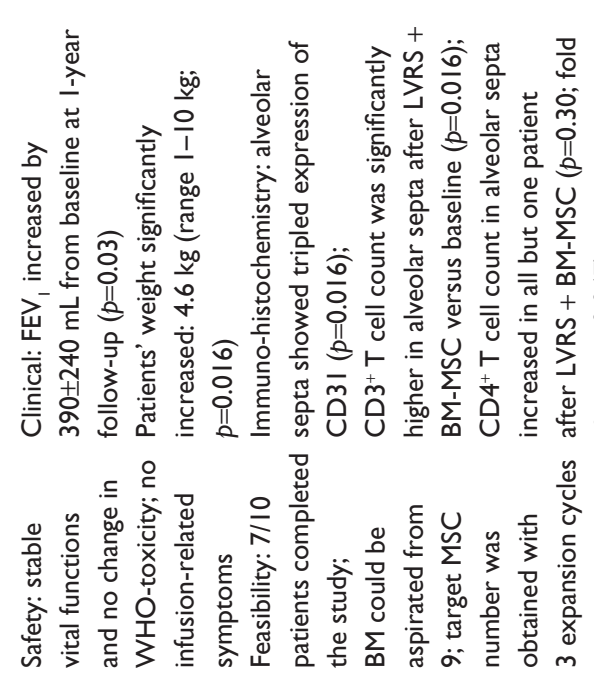

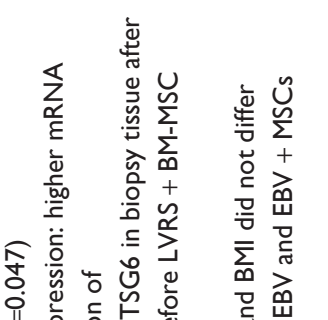

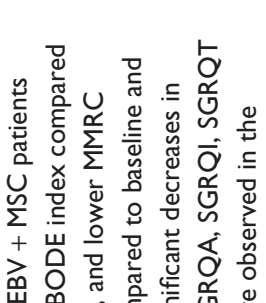

.

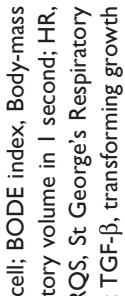

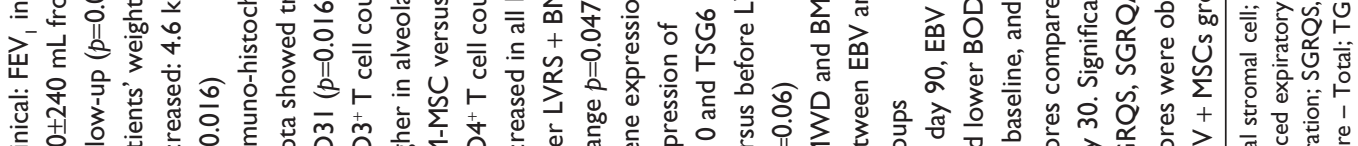

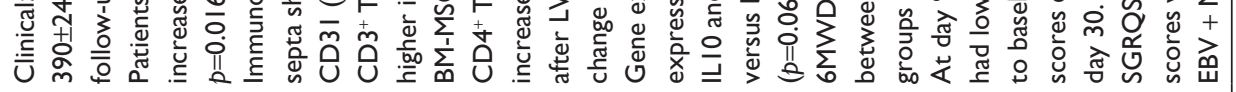

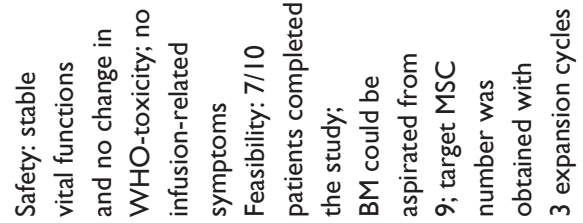
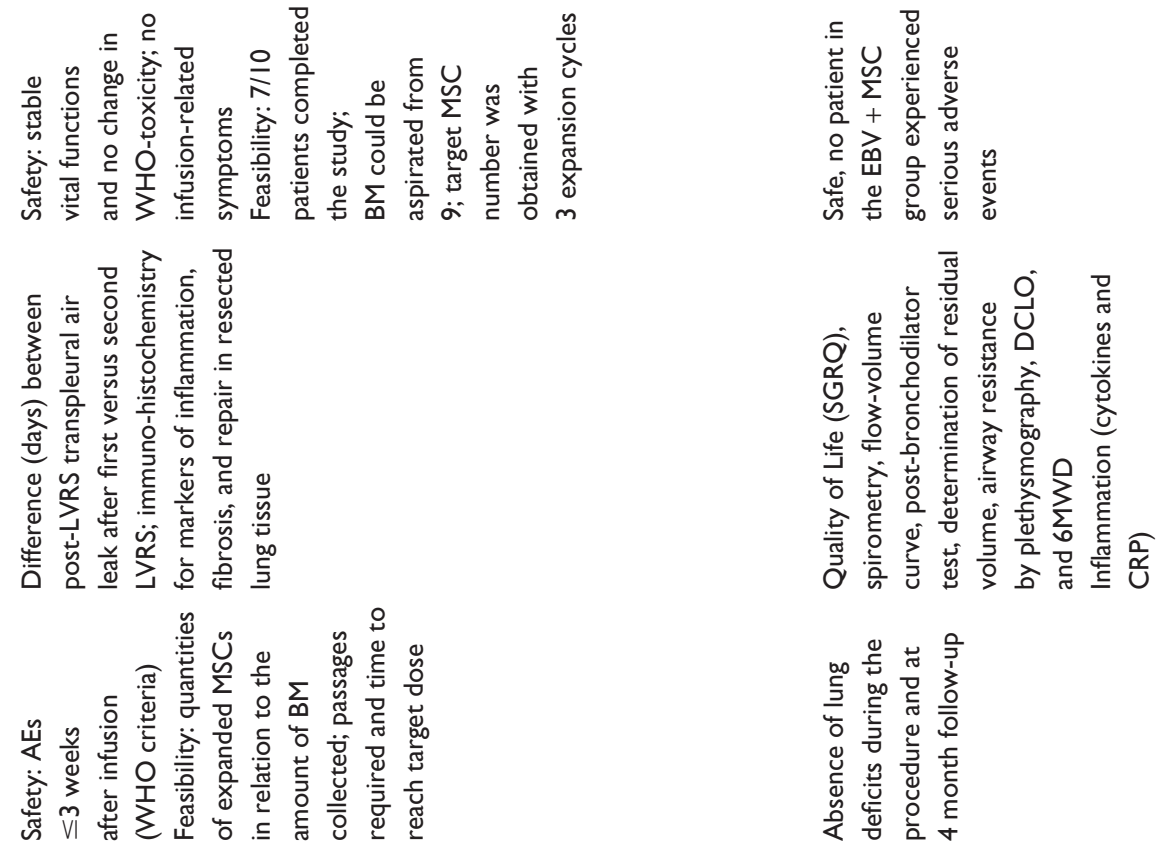

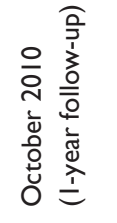
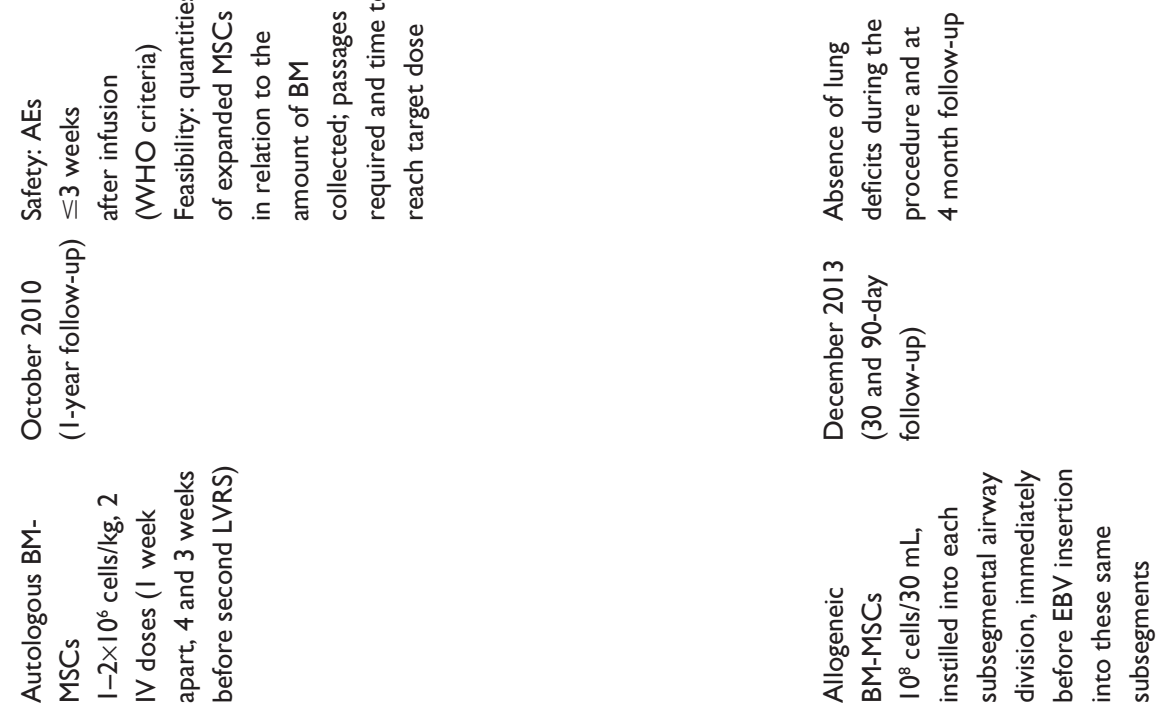

焉离高

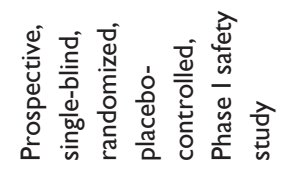

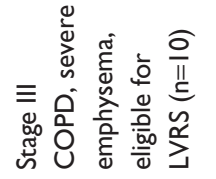

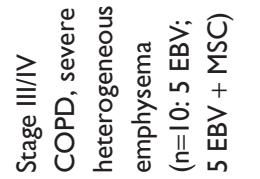

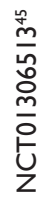

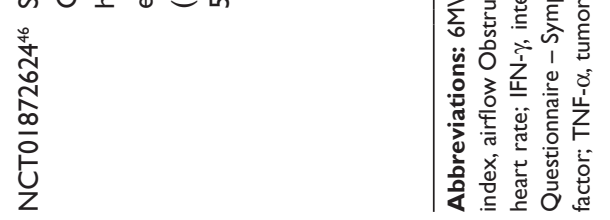


cells are easier to obtain (in a less invasive procedure) and available in higher numbers, which facilitates autologous transplantation. However, no clinical results of adiposederived stem cell therapy in COPD have been published so far. This section of the review will focus on discussing the 4 completed and published clinical trials (NCT00683722, NCT01110252, NCT01306513, and NCT01872624).

A Brazilian Phase I clinical trial employing autologous bone marrow mononuclear cells (BM-MCs) in COPD was carried out by the Genetic and Cell Therapy Laboratory of Universidade Estadual Paulista (São Paulo) in collaboration with the Instituto de Moléstias Cardiovasculares. This study was conducted from May to October 2009 in 4 patients with advanced (GOLD IV) COPD, as approved by the Brazilian National Research Ethics Committee (CONEP). All patients received granulocyte colony-stimulating factor for 3 consecutive days to increase the number of stromal cells in the BM. BM-MCs (which include BM-MSCs) were slowly infused into the medial brachial vein, immediately after preparation $(30 \mathrm{~mL}$ of cell suspension at a concentration of about $1 \times 10^{8}$ cells $/ \mathrm{kg}$ ). Clinical and laboratory follow-ups were carried out in 6 scheduled appointments over 12 months following the procedure. In one of the patients, long-term progress was severely hampered by several complications not related to the procedure, and he died of a hospitalacquired infection $\sim 1$ year later. A second publication reported follow-up of the remaining patients up to 3 years. ${ }^{44}$ One year following BM-MC treatment, patients underwent clinical evaluations using spirometry (with bronchodilator challenges) to attest the maintenance of pulmonary function. Although the small sample size precludes a reliable statistical analysis, this trial was the first to demonstrate the safety and efficacy of BM-MC harvesting and infusion in COPD patients, without any adverse effects. ${ }^{26}$

In the USA, a prospective, randomized, double-blind, placebo-controlled trial was conducted in 62 patients with moderate-to-severe COPD (GOLD II or III) from 6 different centers. ${ }^{27}$ Patients were randomly divided to receive either non-human leukocyte antigen-matched allogeneic MSCs (PROCHYMAL ${ }^{\circledR}$; Osiris Therapeutics, Inc., Columbia, MD, USA) or placebo (vehicle). Patients received 4 monthly infusions $\left(100 \times 10^{6} \mathrm{MSCs} /\right.$ infusion $)$ and were subsequently followed for 2 years after the first PROCHYMAL infusion. Endpoints included comprehensive safety evaluation, pulmonary function testing (spirometry, total lung capacity by plethysmography, single-breath carbon monoxide diffusing capacity, exercise performance [6-min walk test], and dyspnea assessment [Borg scale]), quality-of-life indicators, including questionnaires (St George's Respiratory Questionnaire), and assessments of systemic inflammation (circulating levels of inflammatory mediators). Overall, 19 of 30 patients in the MSC group and 27 of 32 in the placebo group completed the full protocol. Although 27 patients (90\%) in the MSC group and 28 patients (87.5\%) in the placebo group experienced an adverse event over the study duration, no severe or fatal adverse events were associated with PROCHYMAL infusion. There were no significant differences in pulmonary function or quality-of-life indicators; however, an early ( 1 month after injection) and significant decrease in circulating levels of $\mathrm{C}$-reactive protein (CRP) was observed in patients in the MSC group who had high CRP levels at study enrollment. ${ }^{27}$

In the Netherlands, a Phase I, open-label prospective study was conducted to assess the safety and feasibility of intravenous administration of BM-MSCs as an adjuvant therapy in 7 patients with severe emphysema. ${ }^{45}$ In this trial, eligible patients had lung volume reduction surgery (LVRS) at 2 different time points. During the first LVRS, BM was collected from the posterior iliac crest; MSCs were isolated from this tissue and expanded in vitro. Eight weeks later,

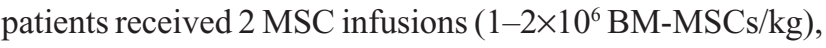
1 week apart, followed by the second LVRS procedure 3 weeks after the second BM-MSC infusion. Adverse events were monitored during the first 3 weeks after infusion, whereas spirometry, gas transfer, lung volumes, and computer tomography-derived lung densitometry were measured at baseline and at 12-month follow-up. No adverse events related to MSC infusions occurred, and lung tissue showed no fibrotic responses. Combined treatment with LVRS and MSCs led to a 3-fold increase in expression of the endothelial marker CD31, as well as $\mathrm{CD}^{+}$and $\mathrm{CD}^{+} \mathrm{T}$ cells in alveolar septa. One year after treatments, pulmonary function tests revealed a significant increase in the forced expiratory volume in 1 second $\left(\mathrm{FEV}_{1}\right)$ of $390 \pm 240 \mathrm{~mL}(p=0.03)$, while residual volume decreased to $540 \pm 145 \mathrm{~mL}(p=0.053)$ compared with baseline. However, LVRS itself has a substantial effect on $\mathrm{FEV}_{1}{ }_{1}{ }^{45}$ which likely could not be further improved by the BM-MSCs.

More recently, a Phase I, prospective, single-blind, randomized, placebo-controlled study was performed in 10 patients with heterogeneous advanced emphysema (GOLD III or IV) from Hospital de Clínicas de Porto Alegre, Brazil. ${ }^{46}$ As in the Dutch trial, this study aimed to evaluate the impact of BM-MSCs as an adjuvant therapy. One-way endobronchial valve (EBV) insertion was combined with intratracheal administration of MSCs. ${ }^{46}$ Patients were 
randomly divided to receive either allogeneic $\mathrm{BM}$-derived MSCs ( $10^{8}$ cells, EBV+MSC) or $0.9 \%$ saline solution (EBV) ( $n=5$ per group), bronchoscopically, just before placement of one-way EBVs. All patients completed the protocol and 90-day follow-up (evaluations on days 0, 1, 7, 30, and 90) after therapy. EBV was well tolerated by all patients but one developed pneumothorax, pneumonia, empyema, and respiratory failure, resulting in removal of all valves. MSC administration was well tolerated, and no serious or clinically significant symptoms or signs were observed during instillation. There were no significant between-group differences in overall number of adverse events, frequency of COPD exacerbations, or worsening, as well as no significant differences in blood tests, pulmonary function tests, or radiological outcomes. The combination of EBVs and MSCs was safe and not associated with any direct adverse event. Serial toxicologic outcomes did not change significantly between the 2 groups and did not change significantly over time. Although circulating CRP levels did not differ significantly between groups at baseline or on days 1 and 7, there was a significant late reduction in CRP levels in EBV+MSC compared with EBV (days 30 and 90). EBV+MSC patients had evidence of increased quality of life from baseline to day 90, including a lower BODE (Body mass index, airway Obstruction, Dyspnea, and Exercise index) and less functional dyspnea, as assessed by the Modified Medical Research Council questionnaire.

\section{Conclusion}

Over the last decade, an increasing number of preclinical studies demonstrated that MSC administration may prevent or treat experimental COPD/emphysema. Preclinical data have suggested that MSCs are able to revert or mitigate either the inflammatory or fibrogenic processes associated with emphysema, including reduction of neutrophil infiltration, modulation of macrophage polarization, reduction of epithelial/endothelial apoptosis, modulation of inflammatory/ anti-inflammatory mediators, reduction of collagen deposition, reduction of cardiovascular repercussions, and improvement of lung function. Based on these preclinical findings, clinical trials have been initiated to test the safety and efficacy of MSCs (or BM-MCs) in COPD.

Current clinical trials to test MSC treatment in COPD have covered a wide range of factors (stages of COPD, route of administration, cell type, number of injected cells, frequency of treatment, etc.), which may influence the expected outcomes and hinder any comparison between studies. Although many rough edges still need to be smoothed out to determine the perfect therapeutic protocol, it is clear that the outcomes achieved in the clinical trials published to date fall far short of the promises launched by preclinical studies involving MSCs in experimental models of emphysema. MSCs have demonstrated a good "adjuvant" role in the clinical scenario. Trials that used MSCs combined with another, primary treatment (eg, LVRS, EBVs) found that patients derived greater benefit in pulmonary function tests and/or quality of life reports, as well as reductions in systemic markers of inflammation (CRP) and modulation of immune cells ( $\mathrm{T}$ cells).

We know which questions need to be answered in order to improve the efficacy of MSC therapy in COPD/emphysema. Further studies are needed to address these questions and allow potentiation and adjust MSCs effects in this complex and challenging disease.

\section{Acknowledgments}

The authors would like to express their gratitude to Mrs Moira Elizabeth Schöttler and Mr Filippe Vasconcellos for their assistance in editing the manuscript. This study was supported by the Carlos Chagas Filho Rio de Janeiro State Research Foundation (FAPERJ; grant number E-26/103.118/2014), Rio de Janeiro, Brazil; and the Brazilian Council for Scientific and Technological Development (CNPq; grant numbers 469716/2014-2, 465064/2014-0, 400462/2014-1, and 465656/2014-5 [INCT-REGENERA] to PRMR), Brasília, Brazil.

\section{Disclosure}

The authors report no conflicts of interest in this work.

\section{References}

1. Global Strategy for the Diagnosis, Management and Prevention of COPD, Global Initiative for Chronic Obstructive Lung Disease (GOLD); 2017. Available from: http://www.goldcopd.org/. Accessed August 7, 2017.

2. Lozano R, Naghavi M, Foreman K, et al. Global and regional mortality from 235 causes of death for 20 age groups in 1990 and 2010: a systematic analysis for the Global Burden of Disease Study 2010. Lancet. 2012;380(9859):2095-2128.

3. Mathers CD, Loncar D. Projections of global mortality and burden of disease from 2002 to 2030. PLoS Med. 2006;3(11):e442.

4. Antunes MA, Laffey JG, Pelosi P, Rocco PR. Mesenchymal stem cell trials for pulmonary diseases. J Cell Biochem. 2014;115(6):1023-1032.

5. Antunes MA, Abreu SC, Cruz FF, et al. Effects of different mesenchymal stromal cell sources and delivery routes in experimental emphysema. Respir Res. 2014;15(1):118.

6. Abreu SC, Antunes MA, Xisto DG, et al. Bone marrow, adipose, and lung tissue-derived murine mesenchymal stromal cells release different mediators and differentially affect airway and lung parenchyma in experimental asthma. Stem Cells Transl Med. 2017;6(6):1557-1567.

7. Cruz FF, Weiss DJ, Rocco PR. Prospects and progress in cell therapy for acute respiratory distress syndrome. Expert Opin Biol Ther. 2016; 16(11):1353-1360. 
8. Huleihel L, Sellares J, Cardenes N, et al. Modified mesenchymal stem cells using miRNA transduction alter lung injury in a bleomycin model. Am J Physiol Lung Cell Mol Physiol. 2017;313(1):L92-L103.

9. Lee H, Kim KC, Choi SJ, Hong YM. Optimal dose and timing of umbilical stem cells treatment in pulmonary arterial hypertensive rats. Yonsei Med J. 2017;58(3):570-580.

10. Shigemura N, Okumura M, Mizuno S, Imanishi Y, Nakamura T, Sawa Y. Autologous transplantation of adipose tissue-derived stromal cells ameliorates pulmonary emphysema. Am J Transplant. 2006;6(11): 2592-2600.

11. Cho RJ, Kim YS, Kim JY, Oh YM. Human adipose-derived mesenchymal stem cell spheroids improve recovery in a mouse model of elastase-induced emphysema. BMB Rep. 2017;50(2):79-84.

12. Hong Y, Kim YS, Hong SH, Oh YM. Therapeutic effects of adiposederived stem cells pretreated with pioglitazone in an emphysema mouse model. Exp Mol Med. 2016;48(10):e266.

13. Kim YS, Kim JY, Shin DM, Huh JW, Lee SW, Oh YM. Tracking intravenous adipose-derived mesenchymal stem cells in a model of elastase-induced emphysema. Tuberc Respir Dis (Seoul). 2014;77(3): $116-123$.

14. Ingenito EP, Tsai L, Murthy S, Tyagi S, Mazan M, Hoffman A. Autologous lung-derived mesenchymal stem cell transplantation in experimental emphysema. Cell Transplant. 2012;21(1):175-189.

15. Kim YS, Kim JY, Huh JW, Lee SW, Choi SJ, Oh YM. The therapeutic effects of optimal dose of mesenchymal stem cells in a murine model of an elastase induced-emphysema. Tuberc Respir Dis (Seoul). 2015;78(3):239-245.

16. Peron JP, de Brito AA, Pelatti M, et al. Human tubal-derived mesenchymal stromal cells associated with low level laser therapy significantly reduces cigarette smoke-induced COPD in C57BL/6 mice. PLoS One. 2015;10(8):e0136942.

17. $\mathrm{Li} \mathrm{Y}, \mathrm{Gu} \mathrm{C}, \mathrm{Xu} \mathrm{W}$, et al. Therapeutic effects of amniotic fluid-derived mesenchymal stromal cells on lung injury in rats with emphysema. Respir Res. 2014;15(1):120.

18. Chen YB, Lan YW, Chen LG, et al. Mesenchymal stem cell-based HSP70 promoter-driven VEGFA induction by resveratrol alleviates elastase-induced emphysema in a mouse model. Cell Stress Chaperones. 2015;20(6):979-989.

19. Gu W, Song L, Li XM, Wang D, Guo XJ, Xu WG. Mesenchymal stem cells alleviate airway inflammation and emphysema in COPD through down-regulation of cyclooxygenase-2 via p38 and ERK MAPK pathways. Sci Rep. 2015;5:8733.

20. Huh JW, Kim SY, Lee JH, et al. Bone marrow cells repair cigarette smoke-induced emphysema in rats. Am J Physiol Lung Cell Mol Physiol. 2011;301(3):L255-L266.

21. Tibboel J, Keijzer R, Reiss I, de Jongste JC, Post M. Intravenous and intratracheal mesenchymal stromal cell injection in a mouse model of pulmonary emphysema. COPD. 2014;11(3):310-318.

22. Ostanin AA, Petrovskii YL, Shevela EY, Chernykh ER. Multiplex analysis of cytokines, chemokines, growth factors, MMP-9 and TIMP-1 produced by human bone marrow, adipose tissue, and placental mesenchymal stromal cells. Bull Exp Biol Med. 2011;151(1): 133-141.

23. Ricciardi M, Malpeli G, Bifari F, et al. Comparison of epithelial differentiation and immune regulatory properties of mesenchymal stromal cells derived from human lung and bone marrow. PLoS One. 2012;7(5):e35639.

24. Ragni E, Montemurro T, Montelatici E, et al. Differential microRNA signature of human mesenchymal stem cells from different sources reveals an "environmental-niche memory" for bone marrow stem cells. Exp Cell Res. 2013;319(10):1562-1574.

25. Schweitzer KS, Johnstone BH, Garrison J, et al. Adipose stem cell treatment in mice attenuates lung and systemic injury induced by cigarette smoking. Am J Respir Crit Care Med. 2011;183(2):215-225.

26. Ribeiro-Paes JT, Bilaqui A, Greco OT, et al. Unicentric study of cell therapy in chronic obstructive pulmonary disease/pulmonary emphysema. Int J Chron Obstruct Pulmon Dis. 2011;6:63-71.
27. Weiss DJ, Casaburi R, Flannery R, LeRoux-Williams M, Tashkin DP. A placebo-controlled, randomized trial of mesenchymal stem cells in COPD. Chest. 2013;143(6):1590-1598.

28. Shinmura D, Togashi I, Miyoshi S, et al. Pretreatment of human mesenchymal stem cells with pioglitazone improved efficiency of cardiomyogenic transdifferentiation and cardiac function. Stem Cells. 2011;29(2):357-366.

29. Eduardo Fde P, Bueno DF, de Freitas PM, et al. Stem cell proliferation under low intensity laser irradiation: a preliminary study. Lasers Surg Med. 2008;40(6):433-438.

30. de Lima FM, Aimbire F, Miranda H, Vieira RP, de Oliveira AP, Albertini R. Low-level laser therapy attenuates the myeloperoxidase activity and inflammatory mediator generation in lung inflammation induced by gut ischemia and reperfusion: a dose-response study. J Lasers Med Sci. 2014;5(2):63-70.

31. Yamaguchi Y, Ohno J, Sato A, Kido H, Fukushima T. Mesenchymal stem cell spheroids exhibit enhanced in-vitro and in-vivo osteoregenerative potential. BMC Biotechnol. 2014;14(1):105.

32. Lee RH, Pulin AA, Seo MJ, et al. Intravenous hMSCs improve myocardial infarction in mice because cells embolized in lung are activated to secrete the anti-inflammatory protein TSG-6. Cell Stem Cell. 2009;5(1):54-63.

33. Murphy MB, Moncivais K, Caplan AI. Mesenchymal stem cells: environmentally responsive therapeutics for regenerative medicine. Exp Mol Med. 2013;45(11):e54.

34. Kim YS, Kokturk N, Kim JY, et al. Gene profiles in a smoke-induced COPD mouse lung model following treatment with mesenchymal stem cells. Mol Cells. 2016;39(10):728-733.

35. Kennelly H, Mahon BP, English K. Human mesenchymal stromal cells exert HGF dependent cytoprotective effects in a human relevant pre-clinical model of COPD. Sci Rep. 2016;6:38207.

36. Guan XJ, Song L, Han FF, et al. Mesenchymal stem cells protect cigarette smoke-damaged lung and pulmonary function partly via VEGF-VEGF receptors. J Cell Biochem. 2013;114(2): 323-335.

37. Thebaud B, Ladha F, Michelakis ED, et al. Vascular endothelial growth factor gene therapy increases survival, promotes lung angiogenesis, and prevents alveolar damage in hyperoxia-induced lung injury: evidence that angiogenesis participates in alveolarization. Circulation. 2005; 112(16):2477-2486.

38. Moon WS, Rhyu KH, Kang MJ, et al. Overexpression of VEGF and angiopoietin 2: a key to high vascularity of hepatocellular carcinoma? Mod Pathol. 2003;16(6):552-557.

39. Rissanen TT, Rutanen J, Yla-Herttuala S. Gene transfer for therapeutic vascular growth in myocardial and peripheral ischemia. Adv Genet. 2004;52:117-164.

40. Li X, Zhang Y, Yeung SC, et al. Mitochondrial transfer of induced pluripotent stem cell-derived mesenchymal stem cells to airway epithelial cells attenuates cigarette smoke-induced damage. Am J Respir Cell Mol Biol. 2014;51(3):455-465.

41. Liu K, Ji K, Guo L, et al. Mesenchymal stem cells rescue injured endothelial cells in an in vitro ischemia-reperfusion model via tunneling nanotube like structure-mediated mitochondrial transfer. Microvasc Res. 2014;92:10-18.

42. Rustom A, Saffrich R, Markovic I, Walther P, Gerdes HH. Nanotubular highways for intercellular organelle transport. Science. 2004; 303(5660):1007-1010.

43. Spees JL, Olson SD, Whitney MJ, Prockop DJ. Mitochondrial transfer between cells can rescue aerobic respiration. Proc Natl Acad Sci USA. 2006;103(5):1283-1288.

44. Stessuk T, Ruiz MA, Greco OT, Bilaqui A, Ribeiro-Paes MJ, RibeiroPaes JT. Phase I clinical trial of cell therapy in patients with advanced chronic obstructive pulmonary disease: follow-up of up to 3 years. Rev Bras Hematol Hemoter. 2013;35(5):352-357.

45. Stolk J, Broekman W, Mauad T, et al. A phase I study for intravenous autologous mesenchymal stromal cell administration to patients with severe emphysema. QJM. 2016;109(5):331-336. 
46. de Oliveira HG, Cruz FF, Antunes MA, et al. Combined bone marrowderived mesenchymal stromal cell therapy and one-way endobronchial valve placement in patients with pulmonary emphysema: a phase I clinical trial. Stem Cells Transl Med. 2017;6(3):962-969.

47. Zhen G, Liu H, Gu N, Zhang H, Xu Y, Zhang Z. Mesenchymal stem cells transplantation protects against rat pulmonary emphysema. Front Biosci. 2008;13:3415-3422.

48. Zhen G, Xue Z, Zhao J, et al. Mesenchymal stem cell transplantation increases expression of vascular endothelial growth factor in papaininduced emphysematous lungs and inhibits apoptosis of lung cells. Cytotherapy. 2010;12(5):605-614.
49. Katsha AM, Ohkouchi S, Xin H, et al. Paracrine factors of multipotent stromal cells ameliorate lung injury in an elastase-induced emphysema model. Mol Ther. 2011;19(1):196-203.

50. Zhao Y, Xu A, Xu Q, et al. Bone marrow mesenchymal stem cell transplantation for treatment of emphysemic rats. Int J Clin Exp Med. 2014;7(4):968-972.

International Journal of COPD

\section{Publish your work in this journal}

The International Journal of COPD is an international, peer-reviewed journal of therapeutics and pharmacology focusing on concise rapid reporting of clinical studies and reviews in COPD. Special focus is given to the pathophysiological processes underlying the disease, intervention programs, patient focused education, and self management protocols.

\section{Dovepress}

This journal is indexed on PubMed Central, MedLine and CAS. The manuscript management system is completely online and includes a very quick and fair peer-review system, which is all easy to use. Visit http://www.dovepress.com/testimonials.php to read real quotes from published authors.

Submit your manuscript here: http://www.dovepress.com/international-journal-of-chronic-obstructive-pulmonary-disease-journal 